\title{
User Location Forecasting at Points of Interest
}

\author{
Jorge Alvarez-Lozano, J. Antonio \\ García-Macías \\ Computer Science Department \\ CICESE Research Center \\ Ensenada, Baja California, México \\ \{jalvarez,jagm\}@cicese.mx
}

\author{
Edgar Chávez \\ Universidad Michoacana \\ Morelia, Michoacán, México \\ elchavez@fismat.umich.mx
}

\begin{abstract}
Predicting the location of a mobile user in the near future can be used for a very large number of user-centered or crowd-centered ubiquitous applications. It is convenient for the discussion to think in terms of discrete locations driven by Points of Interest (POI) instead of absolute positions. We postulate that POI sequences are Markovian once the data is clustered by day of the week and time of the day. To prove our hypothesis we used a public dataset, used in a previous work [15]. In that paper the authors were able to predict the location of a user with $90 \%$ to $70 \%$ accuracy in five minutes and one hour time windows, respectively. With our approach, using Hidden Markov Models, we are able to predict the next POIs within seven hours without significant accuracy decrease. This result enables a large number of potential applications where the aggregate data of a single users conform the behavior of the crowd.
\end{abstract}

\section{Keywords}

Location forecasting, user mobility, Internet user connections.

\section{Categories and Subject Descriptors}

G.3 [Mathematics of Computing]: Markov processes; C.2.1 [Computer-communication networks]: General

\section{General Terms}

Algorithms, Experimentation, Theory.

\section{INTRODUCTION}

Many everyday tasks depend on deploying resources according to the number of users at a given time in a given location. A quick example is the number of cashiers to be deployed in a supermarket or bank. The traditional way to forecast the resource deployment is by taking local statistics of the requested amount of resources. While this approach is valid, the use of smartphones as a pervasive way to establish pres- ence ${ }^{1}$ may allow to switch the perspective of the problem. Local statistics can be substituted by forecasting the location of a very large sample of users within a few hours time span. Each user data is mined to extract mobility patterns. And as a byproduct it is possible to predict the capacity request for a particular resource. Furthermore, local statistics and crowd location forecasting methods can be combined to increase the accuracy of the prediction.

Mining individual mobility patterns allows to serve various applications. The general public (including banks, retail stores or traffic authorities) could query a location forecasting service to know the capacity of a specific Point of Interest (POI). Relevant examples of usage include traffic shaping when a blockage or detour suddenly appears. Current traffic applications make use of the online updates of the position of the users, but are unable to anticipate an overcrowding highway. In one application scenario, a user may query the capacity of a given POI (think for example in a fast food location) and decide between many different alternatives based on that measure.

A more specialized service can warn the user about a potential overcrowded POI he is about to visit in the next few hours. This is a potential paradox, since all users using the service may back off and avoid the POI, which will not be overcrowded at the end. This may have the same effect of trying to predict the behavior of an individual stock option, any successful forecasting algorithm can change the behavior of a system. For the time being we will focus on forecasting the user location without worrying about paradoxical behavior.

Users should be assured about the potential usage of their data. Disclosing their current location implies they are disclosing travel patterns and the individual forecast of their location. The service should provide secure ways to disclose the location of anonymous users, since the objective is to infer the location of a crowd in a given POI.

We are not aware of public repositories of user location logs for general purpose applications. Since we need to benchmark our approach, we selected Internet user connections as an example because there is a large body of user location

\footnotetext{
${ }^{1}$ As of February 2012 nearly half of the american adults use an smartphone ( http://pewinternet.org/ / media//Files/Reports/2012/Smartphone \\%20ownership \ $\% 202012$.pdf)
} 
data is available for benchmarking. The results of the application predict the location of a single user at a given time, it is not difficult to see that we can predict how many users will be in a particular POI at a given time. Predicting the location of every user in the system is a way to estimate how many users will be in every POI.

The rest of this paper is organized as follows: we discuss with some detail the related work in spatio-temporal predictions. After that, we describe our approach which is based on clustering the user data by periods of time, we postulate that this clustering makes the sequence of user positions a Markovian chain; hence we use Hidden Markov Models for the forecasting part. Next, we experimentally validate our approach using a public dataset. Finally, we discuss the scope and limitation of our approach and present some conclusions.

\section{RELATED WORK}

Mobility prediction, mining or forecasting have many different sources depending on the application. Some works try to characterize the activities of a user, to obtain behavior patterns and predict mobility as in $[14,5,13]$. One particular approach in [15] is general enough to be significant to compare with our approach. The idea is to perform mobility forecasting by identifying significant locations, in particular locations with WiFi access. Access points have the characteristics of a POI. It is important to notice, however, that a user may not connect to a hotspot and may still be there. Hence we need to take this data with caution. The authors of [15] get as reference the sequence of the places visited by a user, and using the historical records, they search for a similar pattern in the past to predict the next place to visit and the time the user will stay there. The best precision (about 90\%) is obtained when the prediction period is only about 5 minutes, if the prediction time increases to 60 minutes the precision drops to $70 \%$. We selected the data, which is publicly available, to test our proposal; we compared with the results of this paper and improved the prediction significantly.

In some application scenarios of position forecasting, the user can transit in a discrete network. From a given cell the user can reach a limited number of cells. This happens for example in the cellular network, a user moving from one cell to another, can benefit from some caching in the data transmission if the next cell to be visited can be anticipated. Assuming the user does not suddenly disappear, she will leave one cell and connect only to one of the neighboring cells of the current position. This approach is explored in [7] and the authors use some machine learning approaches to deduct the next cell of the user. As it can be seen, this is a restricted version of the problem stated.

\section{USER MOBILITY}

People have recurrent mobility patterns, it is rare to have a completely erratic behavior over the time. We normally have routines which can vary over time, but within certain behavior boundaries. We can distinguish between weekday, weekend, monthly or annually patterns. This is analyzed in $[14,16]$. Once we recognize the user mobility pattern, we are enabled to predict her position in time and space. In this work we have fixed our attention in three major features of

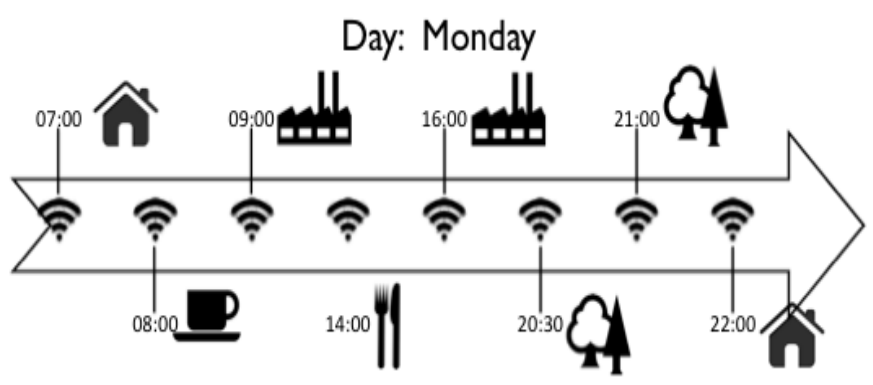

Figure 1: The next user location depends only on the current location, once the sequences have been factorized by week day and time of the day.

user mobility and considering their interrelation we can infer user mobility (Figure 1).

\subsection{Temporal Patterns}

It is reasonable to assume certain periodicity in location/time patterns. Usually weekdays are similar, people tend to organize their life according to working hours and our hypothesis is that activities in the same weekday will have a repetitive pattern. A corresponding periodicity is observed during weekends. The places visited during weekdays at the same hour are postulated to be the same. We will group the historic data by weekdays, weekends and hours.

One additional observation is that the current location in a given day and hour, conditions the next place to be visited. For example if one user is at home at 7:00 AM on a Monday, the next place he will be at is most likely the coffee shop or the office, but not the restaurant or a movie theater. This can be described with a Markov chain. The main property, the Markovian property [2], is that the current place is only a function of the previous place. Our main claim is that once the data is grouped by the above temporal slices (weekdays and weekends, hours and minutes) the sequence of places visited form a Markovian chain.

\section{CONNECTIVITY PREDICTION}

In order to make spatio-temporal predictions of Internet user connections, we consider three features for mobility (week day, time of the day, and current location) and the relationships between them. Accordingly, if during the last 4 Mondays the user has connected at home at 07:00, then at 09:00 at her office, then at 14:00 at a restaurant, and so on, until connecting again at home at 22:00, it could be determined with a certain probability that the user will follow the same sequence of connections next Monday (Figure 1). That is, next Monday she will probably connect at home at 07:00, and then according to the observed transition on the previous Mondays, it is probable that she will next connect at the office around 09:00.

Several methods have been proposed to identify places that have significance for a user [5][9][4]. We should note that these places are usually called significant places in the literature, although in this paper we will refer to them as points of interest (or abbreviated as POI). In some previous works with similar applications, the POIs have been identi- 
fied using GPS records [13],[9]. Locating POI's with a GPS makes a lot of sense for our application, unfortunately the data used in those papers is not available. We will use the publicly available WiFi access points records of [15] for our work. Therefore, we consider points of interest those providing Internet connectivity, which the user visits frequently and spends some time at; a place is considered significant if the user spends at least $t$ seconds there on each visit, and he has visited it at least $n$ times

User mobility may vary over time and thus the places visited. Places that some months ago were frequented by a user, may eventually cease to be of inters. For instance, the significant places for students differ when there is a school period, spring break, or winter vacations. Therefore, we applied the two metrics to the records of different periods of time (one, two and three months). In the next section we present the results using the presented metrics.

\subsection{Our concrete experiment}

A Hidden Markov Model (HMM) [11] is a finite state machine consisting of a set of hidden states $(Q)$, a set of observations $(O)$, transition probabilities $(A)$, emission probabilities $(B)$, and initial probabilities for each state $(\pi)$. Hidden states are not directly visible, although observations are visible. Each state has a probability distribution over the set of observations. Hence, if we consider a sequence of observations it would be possible to determine the sequence of hidden states that generated it (Figure 2).

Taking the above into account, if we have knowledge about the day of the week, the transition of the user between the POIs, and the probability of a user being connected at a given place at a given time, it is feasible to predict in which POIs a user will be connected at a given time period. For instance, if we know that the connection sequences for a user in the last 3 Mondays were: at places defined as $q_{1}, q_{2}$, and $q_{4}$ respectively, at 07:00, 09:20, and 14:30; in $q_{1}, q_{2}$, and $q_{4}$, at 07:10, 09:05, and 14:08; and finally in $q_{1}, q_{2}$, and $q_{4}$ at 07:15, $09: 10, y 14: 17$, then it is possible to infer the sequence for the following connections. Therefore, in order to predict the connections that will take place next Monday in the 08:30 to 15:00 time period, we will consider the connections that took place the preceding Mondays in the same time period, the places where they were made, and the mobility of the user between those places. This way, there would be a certain probability of the resulting sequence being at places: $q_{2}$ and $q_{4}$, with approximate connection times of 09:15 and 14:00, respectively.

\section{OUR MODEL AND EXPERIMENTS IN DETAIL}

\subsection{Dataset}

In order to evaluate our proposal we used Dartmouth's dataset [6]. This dataset contains records of connections of the Dartmouth College Campus, captured over a period of 3 years. One reason for the selection of this dataset is that for each record, it provides date, place (access point) and time of connection. Therefore, it is possible to define the required parameters for the HMM and estimate user mobility between POIs and the associated connection times. Also, this dataset has been used in different published works $[15,13]$ to estimate user mobility, providing a good references for comparison purposes.

\subsection{POI selection}

Even though the selected dataset has a great amount of records for each user, not all of them are useful for our purpose; some of them have short periods of connection. Therefore, we have only considered the records having connection times of more than $t=30$ seconds, this is in order to avoid places that are not important to the users. For instance, when the user passes by a public place with WiFi connectivity, a connection could be automatically made as the wireless interface scans and finds an access point, but this will be a short-lived, involuntary and needless connection. Also, with connections lasting at least 30 seconds, using a $802.11 \mathrm{~g}$ interface, and under an optimistic scenario, it is possible to make transfers of $200 \mathrm{MB}$ approximately. Considering that the WiFi connectivity may be intermittently available, a long time connection can be split in several shorter connections. In order to avoid this problem, and to obtain a more accurate prediction, we merge the records if: given a sequence of records to the same place, the end time of the $x_{i}$ record is close to the start time of the $x_{i+1}$ record. That is, EndTime $_{x_{i}}-$ StartTime $_{x_{i+1}} \leq \delta$. We defined the value of $\delta$ $=300$ seconds, as NextPlace did. After we apply the merge procedure, we select the POIs according to the frequency of visits; we define a place as a POI if the user has visited it at least $n=20$ times.

As it was mentioned before, we are interested in to identify the places that are significant at the time. Therefore, for each user we take as reference the records of the last month, and we apply to them the mentioned metrics. Then we apply the metrics to the records corresponding to the previous one, two, and three months. When considering the records of the previous month we obtain on average 5.83 POIs; in contrast, using records of the previous two and three months, we obtain 8.95 and 9.08 POIs, respectively. Finally, we compare the POIs of the last month with those obtained using the records of the previous one, two and three months. Although, using records of the previous three months we obtained the most number of POIs, the records of the previous two months define in a more accurate way the current POIs for each user. Therefore, the records for the last previous two months are used to define the HMM and thus make the spatio-temporal prediction.

\subsection{Spatio-temporal prediction}

Due to the characteristics considered about the mobility of each user, and the relationship between these characteristics, the connection records (for the last two months) have been grouped according to the day they occurred. This way, we used the records for the previous Mondays (i.e., eight) to make the prediction about the connections sequence for next Monday (i.e., ninth Monday). For each record, the $(d, t, p)$ tuple is defined, where $d$ represents the connection date, $t$ represents the time of the day, and $p$ represents the place of connection. With this information, the HMM is defined as:

Hidden states. Defined by the set of POIs for each user. We have also defined an additional state indicating the lack of Internet connection. For instance, when considering the POIs for Figure 2 (five places), there would be six hidden 


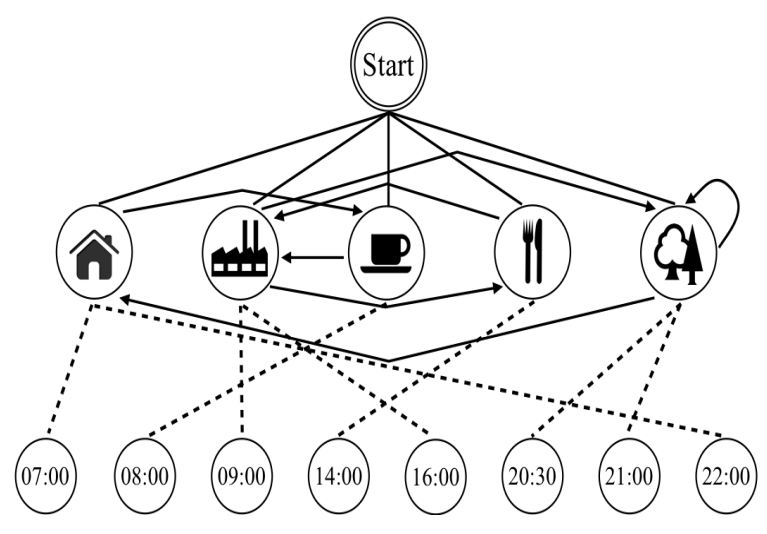

Figure 2: An HMM representing POIs and their relationship with different connection times.

states: $Q=q_{1}, q_{2}, q_{3}, q_{4}, q_{5}, q_{6}$. Where: $q_{1}$ corresponds to home, $q_{2}$ to the office, $\ldots, q_{6}$ to no connection.

Observations. Defined as follows: the time period between the first and last connections is segmented in $m$ intervals, according to the value of $t_{i c}$ (eq. 1). That is, the intercontact times average of Internet connections is obtained. This way, if the times for the first and last connections are 07:00 and 19:00 (720 minutes), with a $t_{i c}$ value of 1:30 hours (90 minutes), then 8 intervals are obtained. Consequently, the observations for this user would be: $O=07: 00,08: 30, \ldots$ , 17:30, 19:00. We decided to use the inter-contact times to define the observations because some previous works have utilized this property to study the human mobility and estimate future contacts[3, 8$]$.

$$
t_{i c}=\text { average (inter-contact times) }
$$

Vector $\pi$. Defines the initial probability for each POI. In this case, it represents the probability of the first connection of the day being to a POI $q_{i}$ (i.e., $\pi=[0.5,0.2,0.1,0.1,0.05,0.05]$ ).

Matrix A. It is defined by analyzing the connections records and calculating the probability of a user moving from a place $q_{i}$ to the remaining POIs (including the no-connection state) for all $1 \leq i \leq N$, or else, to the user staying at the same place. For this work we have considered using first order hidden Markov models, therefore, to define matrix $A$, only the last movement made to arrive to a place $q_{i}$ is considered.

Matrix B. It is defined when determining in what POI $q_{i}$, the user has been connected, given an observation $o_{j}$, for all $1 \leq i \leq N$ and $1 \leq j \leq M$. That is, the user connection probability for each time (defined by observations \pm a range $\epsilon)$ in every POI $q_{i}$.

Using the data in Figure 1, the HMM is defined in Figure 2. Then, the predictions can be made. For the purposes of our work, it is required to know the number of Internet connections the user will make, and at what times they will take place. That is, for a time $T$, we are interested in to know the connections for a given time period $[T, T+\Delta T$ minutes ]. For this, vector $\pi$, matrix $A$, and matrix $B$, are used to identify the combinations of hidden states that satisfy such time period and their corresponding probabilities, so later

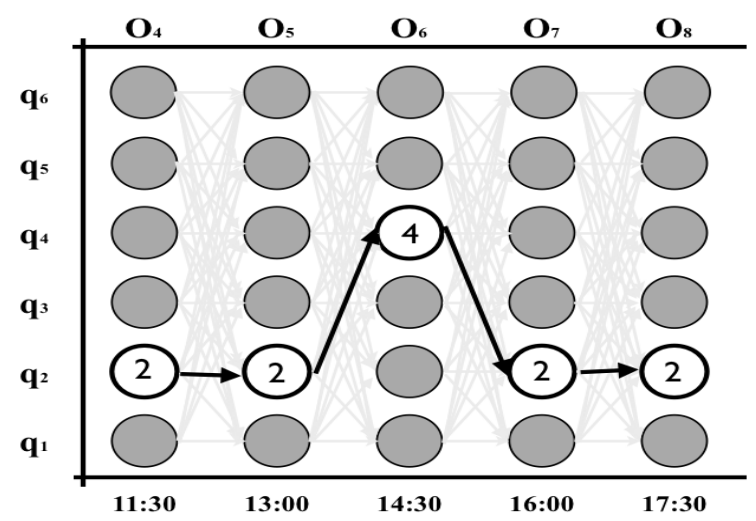

Figure 3: Using the Viterbi algorithm to identify the POIs where the user will have (or not) Internet connection given a period of time.

the sequence of hidden states with the highest probability can be selected. In order to aid in the identification of the sequence of hidden states with the highest probability, we use Viterbi's algorithm [1].

Using the parameters from the HMM, Viterbi's algorithm determines the sequence of hidden states (with the highest probability) that generates a given sequence of observations. For example, suppose that we wish to know the connections that a certain user will have on Monday from 11:00 to 18:00, and the user has a set of observations $O=07: 00,08: 30$, 10:00, 11:30, 13:00, 14:30, 16:00, 17:30, 19:00. In this case, the reference set would be: $O=o_{4}, o_{5}, o_{6}, o_{7}, o_{8}$, corresponding to: 11:30, 13:00, 14:30, 16:00, and 17:30, and as result the best sequence of hidden states that can be generated from this sequence of observations is: $q_{2}, q_{2}, q_{4}, q_{2}, q_{2}$. This means that the user will connect to Internet at 11:30 and 13:00 at the place defined as $q_{2}$, at 14:30 will connect at $q_{4}$, and finally at 16:00 and 17:30 at $q_{2}$ (Figure 3). As we discussed earlier, we are interested in supporting applications where predictions can be made for the next $n$ hours, not only for the next few minutes.

\subsection{Evaluation}

To test the efficacy of our spatio-temporal prediction we compare it against the method presented in NextPlace[15]; their results have been the best in the literature up to the best of our knowledge. However our method estimates the full sequence of POIs where the user will be in a given period of time, while the method presented in NextPlace estimates the place where the user will be at a specific time. For this reason, and to do a fair comparison of the performance of both methods, for each observation in the period of time we apply the best reported method of NextPlace(NP3) to predict where the user will be. That is, if we want to know the sequence of POIs in the $[10: 00,15: 00]$ period, and considering that for this period there are 3 observations defined (11:30, 13:00, and 14:30), we apply the method of NextPlace to each observation (3). Then, we average the results in order to compare with our method.

\section{RESULTS}




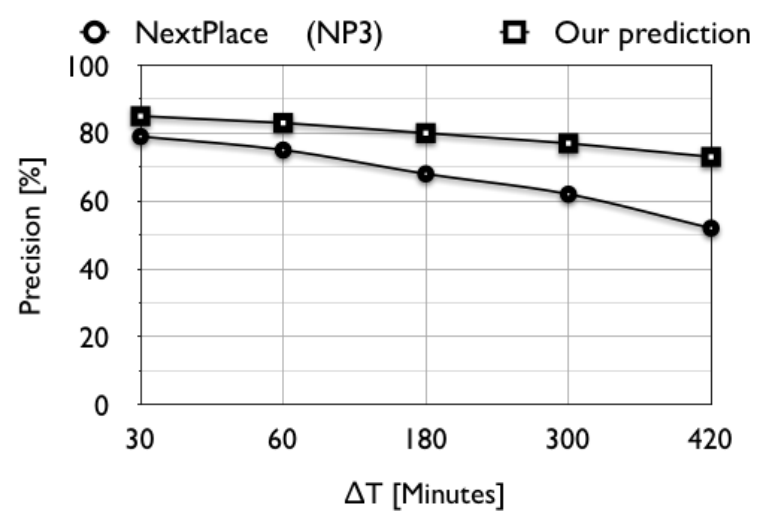

Figure 4: Prediction precision using observations for different values for $\Delta T$.

We show the efficiency of our method by making several predictions involving a set of users. Predictions were made considering records for 200 users. For each one of the 200 users, we use records of the last month to test the prediction, and records of the previous two months are used to identify the POIs and to define the HMM. For each user, we have made five predictions for each day of the week considering different observations corresponding to different values of $\Delta T$ (30 minutes, $1,3,5$, and 7 hours). A total of 7000 predictions, 35 predictions for each user. Each prediction is uniformly distributed over the [07:00,17:00] interval. We selected this interval because in the worst case, if we make a prediction at $T=17: 00$, it is possible to know in which POIs a user will be using the largest value for $\Delta T$ ( 7 hours).

To determine the effectiveness of the prediction, if we estimate where a user will be in the interval $[T, T+\Delta T]$, the prediction is correct if the user is at place $q_{i}$ in the interval $\left[T_{\text {pred }}-\theta, T_{\text {pred }}+\theta\right]($ eq. 2$), \theta$ represents an error margin. That is, a prediction is considered correct when the user is connected at the place $q_{i}$, at the time indicated by the observation $o_{i}$. It would also be correct if the prediction indicates that the user is not connected (in the case of the state corresponding to no connection). We have defined $\theta=$ 10 minutes.

$$
T_{\text {pred }}=T+o_{i} \quad 1 \leq i \leq \text { Number of }
$$$$
\text { observations in the interval }
$$

In Figure 4, we show our results against those obtained using NextPlace. Even though the number of users and the amount of predictions made are small, the preliminary results are encouraging. When considering a period of $30 \mathrm{~min}-$ utes, we obtain better results of up to $85 \%$ precision against $79 \%$ for NextPlace; for a period of 60 minutes, we obtain up to $83 \%$ against $75 \%$ for NextPlace. When the value of $\Delta t$ increases, the precision for NextPlace decreases. For periods longer than 60 minutes, the precision for NextPlace lies within the range of $50 \%-70 \%$. In contrast, with our proposal, we obtain up to $80 \%$ precision for a period of 3 hours; a period of 5 hours yields a precision of up to $77 \%$, and finally a 7 hours period yields a precision of $73 \%$. However, for periods beyond 7 hours, the precision obtained drops down to $50 \%$. The previous evaluation shows that our proposal can yield good precision for time periods of up to 7 hours.
Furthermore, we show that considering the three characteristics of mobility (week day, time of the day, and current location) and their relationship, allow to have a more robust prediction, and better results as consequence. We show that our method is better than the the best method presented by NextPlace.

We have used the records for a specific day from the last two months (e.g., $\approx$ records for last eight Mondays), in order to make the prediction for the next day (ninth Monday). However, when making the prediction for the subsequent day (tenth Monday), the precision decreases, and this decrease is sustained for the successive days (eleventh, twelfth). This implies that to obtain a good prediction for a given day $x_{i}$, it is recommended to consider the records for the previous two months. This observation is valid for this dataset alone, for other datasets some data mining is needed to tune the parameters.

\section{CONCLUSION}

People usually exhibit cyclical patterns in their daily activities. Based on this assumption, in this exploratory work we proposed a scalable method to predict user location, which in turn allows predicting the location of crowds by applying the same algorithm to all the individuals of a crowd. The time to update the forecasting of each user is negligible and also suitable to be implemented in a massively parallel architecture such as the graphics processing unit (GPU) commonly found in smartphones. As a working example, we selected the publicly available data of WiFi connections for a large group of users. Our work empirically proves the Markovian property of the sequence of the user POI tags over time, once they have been factored by day of the week and time of the day. Others have suggested [12] that further improvements in the predictions can be made by considering the last two POI (instead of only one), using a second order HMM; however, we experimented with this approach and the results obtained yielded insignificant improvements (an additional $1 \%$ or $2 \%$ accuracy) not worth the extra overhead.

We are considering different avenues for future work. First, we want to factor out the automata representing the user location for a large group of users, and model the crowd as a single entity. Preliminary findings in this direction point to Partially Observable Markov Decision Processes[18] as the relevant model for crowds in this context. Also, a large scale experimental setup for crowd location forecasting is needed to verify our findings. We have not considered the fact that experiments with crowds should contemplate ways to incentivate individuals to participate; many examples of using incentives $[10,17]$ have been proposed in the literature.

\section{REFERENCES}

[1] Andrew J. Viterbi . A personal history of the Viterbi algorithm. IEEE Signal Processing Magazine, 23(4):120-142, July 2006.

[2] Andrey A. Markov. Theory of Algorithms. Israel Program for Scientific Translations, Bloomington, IN, USA, 1961.

[3] Augustin Chaintreau, Pan Hui, Jon Crowcroft, Christophe Diot, Richard Gass, and James Scott. Impact of human mobility on opportunistic forwarding 
algorithms. IEEE Transactions on Mobile Computing, pages 606-620, 2006.

[4] Changqing Zhou, Dan Frankowski, Pamela Ludford, Shashi Shekhar, and Loren Terveen. Discovering personally meaningful places: An interactive clustering approach. ACM Transactions on Information Systems, 25(3):56-68, July 2007.

[5] Daniel Ashbrook and Thad Starner. Using GPS to learn significant locations and predict movement across multiple users. Personal Ubiquitous Computing, 7(5):275-286, October 2003.

[6] David Kotz, Tristan Henderson, Ilya Abyzov and Jihwang Yeo. CRAWDAD trace dartmouth/campus/movement/infocom04 (v 2004-08-05). Downloaded from http://crawdad.cs.dartmouth.edu/dartmouth/campus /movement/infocom04.

[7] Gökhan Yavas, Dimitrios Katsaros, Özgür Ulusoy, and Yannis Manolopoulos. A data mining approach for location prediction in mobile environments. Data \&6 Knowledge Engineering, 54(2005):121-146, August 2004.

[8] Han Cai and Do Young Eun. Crossing over the bounded domain: from exponential to power-law inter-meeting time in manet. In Proceedings of the 13th annual ACM international conference on Mobile computing and networking (MobiCom'07), pages 159-170. ACM Press, 2007.

[9] Jong Hee Kang, William Welbourne, Benjamin Stewart, and Gaetano Borriello. Extracting places from traces of locations. SIGMOBILE Mobile Computing and Communications Review, 9(3):58-68, July 2005.

[10] Juong-Sik Lee and Baik Hoh. Dynamic pricing incentive for participatory sensing. Pervasive Mobile Computing, 6(6):693-708, December 2010.
[11] Lawrence R. Rabiner. A tutorial on hidden Markov models and selected applications in speech recognition. Proceedings of the IEEE, 77(2):257-286, May 1989.

[12] Libo Song, David Kotz, Ravi Jain, and Xiaoning He. Evaluating location predictors with extensive $\mathrm{Wi}-\mathrm{Fi}$ mobility data. SIGMOBILE Mobile Computing and Communications Review, 7(4):64-65, October 2003.

[13] Minkyong Kim, David Kotz and Songkuk Kim. Extracting a Mobility Model from Real User Traces. In Proceedings of the 25th Annual Joint Conference of the IEEE Computer and Communications Societies (INFOCOM'06), pages 1-13. IEEE, 2006.

[14] Nathan Eagle and Alex (Sandy) Pentland. Reality mining: sensing complex social systems. Personal Ubiquitous Computing, 10(4):255-268, March 2006.

[15] Salvatore Scellato, Mirco Musolesi, Cecilia Mascolo, Vito Latora, and Andrew T. Campbell. NextPlace: a spatio-temporal prediction framework for pervasive systems. In Proceedings of the 9th international conference on Pervasive computing (Pervasive'11), pages 152-169. Springer-Verlag, 2011.

[16] Sara Motahari, Hui Zang, and Phyllis Reuther. The impact of temporal factors on mobility patterns. In Proceedings of the 45th Hawaii International Conference on System Sciences (HICSS'12), pages 5659-5668. IEEE Computer Society, 2012.

[17] Sasank Reddy, Deborah Estrin, Mark Hansen, and Mani Srivastava. Examining micro-payments for participatory sensing data collections. In Proceedings of the 13th international conference on Ubiquitous computing (Ubicomp'10), pages 33-36. ACM Press, 2010.

[18] Weihong Zhang. Algorithms for partially observable Markov decision processes. PhD thesis, Hong Kong University of Science and Technology, 2001. 